\title{
Evidence for Neuronal Desynchrony in the Aged Suprachiasmatic Nucleus Clock
}

\author{
Sahar Farajnia, Stephan Michel, Tom Deboer, Henk Tjebbe vanderLeest, Thijs Houben, Jos H. T. Rohling, \\ Ashna Ramkisoensing, Roman Yasenkov, and Johanna H. Meijer \\ Department of Neurophysiology, Leiden University Medical Center, 2300 RC Leiden, The Netherlands
}

\begin{abstract}
Aging is associated with a deterioration of daily (circadian) rhythms in physiology and behavior. Deficits in the function of the central circadian pacemaker in the suprachiasmatic nucleus (SCN) have been implicated, but the responsible mechanisms have not been clearly delineated. In this report, we characterize the progression of rhythm deterioration in mice to $900 \mathrm{~d}$ of age. Longitudinal behavioral and sleep-wake recordings in up to 30-month-old mice showed strong fragmentation of rhythms, starting at the age of $700 \mathrm{~d}$. Patch-clamp recordings in this age group revealed deficits in membrane properties and GABAergic postsynaptic current amplitude. A selective loss of circadian modulation of fast delayed-rectifier and A-type $\mathrm{K}^{+}$currents was observed. At the tissue level, phase synchrony of SCN neurons was grossly disturbed, with some subpopulations peaking in anti-phase and a reduction in amplitude of the overall multiunit activity rhythm. We propose that aberrant SCN rhythmicity in old animals-with electrophysiological arrhythmia at the single-cell level and phase desynchronization at the network level— can account for defective circadian function with aging.
\end{abstract}

\section{Introduction}

Almost all living systems have developed endogenous circadian clocks with periods of $\sim 24 \mathrm{~h}$ to anticipate changes in the solar day. In mammals, the circadian clock is located in the suprachiasmatic nuclei (SCN) of the anterior hypothalamus (Takahashi et al., 2008). Based on a molecular feedback loop, individual neurons of the SCN produce $24 \mathrm{~h}$ rhythms resulting in autonomous single-cell oscillators (Welsh et al., 1995). Importantly, the circadian pacemaker of the SCN functions as a multi-oscillator structure, and interneuronal coupling is required to obtain sufficient amplitude to drive behavioral activity at one phase of the cycle and to silence the organism at another phase (Takahashi et al., 2008).

With aging, deterioration of circadian rhythms is observed in animals and humans alike. The deterioration is accompanied by a decrease in circadian amplitude, evidenced by sleep loss at night and difficulty staying awake during the day (Turek et al., 1995; Dijk et al., 1999; Hofman and Swaab, 2006). Nearly all measured physiological functions show a decrease in rhythm amplitude, including temperature and melatonin rhythms (Dijk et al., 1999; Mahlberg et al., 2006). Implantation of neonatal SCN tissue into aged hamsters improves circadian rhythmicity and increases lon-

Received Jan. 31, 2012; revised March 2, 2012; accepted March 5, 2012.

Author contributions: S.M., T.D., and J.H.M. designed research; S.F., T.D., H.T.V., T.H., and R.Y. performed research; S.F., T.D., H.T.V., J.H.T.R., and A.R. analyzed data; S.F., S.M., T.D., and J.H.M. wrote the paper.

This research was supported by Netherlands Organization for Scientific Research Grant 818.02.016. We are grateful to Bill Schwartz, Mike Antle, and Eus van Someren for their comments on this manuscript. We thank Jan Janse and Sander van Berloo for electronic assistance and Hans Duindam for technical assistance. We thank Eva Koster and Doortje Krijbolder for their help with the analysis of SCN electrical activity recordings.

Correspondence should be addressed to Johanna H. Meijer, Department of Neurophysiology, Leiden University Medical Center, Einthovenweg 20, P.0. Box 9600, 2300 RC Leiden, The Netherlands. E-mail: j.h.meijer@lumc.nl.

DOI:10.1523/JNEUROSCI.0469-12.2012

Copyright $\odot 2012$ the authors $\quad 0270-6474 / 12 / 325891-09 \$ 15.00 / 0$ gevity (Hurd and Ralph, 1998), indicating that proper functioning of the SCN is crucial for healthy aging.

Several studies have indicated aging-induced cellular changes in the SCN. Among them are a decrease in amplitude of SCN electrical activity rhythms (Satinoff et al., 1993; Watanabe et al., 1995 ) and a reduction in peptidergic function, including vasoactive intestinal peptide (VIP), gastrin releasing peptide, neurotensin, and vasopressin (Swaab et al., 1985; Harper et al., 2008; Duncan et al., 2010). Alterations in the density of GABAergic axon terminals and in the $\alpha 3$ subunit of the $\mathrm{GABA}_{\mathrm{A}}$ receptor have been reported (Palomba et al., 2008) as well as changes in melatonin receptors (Wu et al., 2007). In other parts of the nervous system, age-related behavioral changes were paralleled by altered membrane properties (Luebke et al., 2010), but few studies have investigated the membrane function in the aged SCN (Nygård et al., 2005).

Given the observed cellular changes, it is possible that single-cell membrane properties are compromised in SCN neurons of old animals. Alternatively, aging may primarily affect the SCN network organization, leading to a phase desynchronization among clock neurons. The latter hypothesis is in accordance with recent indications that reduced synchronization is a common feature of aging neuronal networks (Stam et al., 2005; Smit et al., 2010). To test this hypothesis, we have analyzed circadian rhythms in the course of aging on different levels of organization, from single-cell properties to overt behavior and sleep-wake regulation. In longitudinal behavioral and sleep recording experiments in mice, we studied the changes in activity and sleep parameters as a function of age. Recordings of neuronal subpopulations in $\mathrm{SCN}$ slices of young and old mice elucidated that aging had affected the SCN network-level organization. Patch-clamp recordings were performed to determine whether the changes in the network were explainable by alterations of singlecell attributes. 


\section{Materials and Methods}

Behavioral activity recordings

Male C57BL/6 (Harlan) were obtained and housed under a $12 \mathrm{~h}$ light/ dark cycle in sound-attenuated, climate-controlled rooms with ad libitum access to food and water. Animals were individually housed in cages with a running wheel to record their behavioral activity pattern. The number of wheel revolutions was digitized in $1 \mathrm{~min}$ bins with a computerized recording system (Clocklab; Actimetrics). At different ages along the entire lifespan of the animals, lights were turned off for a minimum of $10 \mathrm{~d}$ to assess the properties of the circadian pattern of behavioral activity in constant darkness (DD). Behavioral activity was plotted in actograms, and the circadian period $(\tau)$, active period $(\alpha)$, and resting period $(\rho)$ were determined using the Clocklab analysis software. The amount of wheel running and the duration of wheel running bouts was calculated using Igor Pro (Wavemetrics). Longitudinal data obtained under the $12 \mathrm{~h}$ light/dark cycle were used to visualize the distribution of activity throughout the day, perform an F-periodogram analysis, and quantify the intradaily variability (IV) (Witting et al., 1990). Phase-shifting capacity was tested in young and old animals by exposing them to a $30 \mathrm{~min}$ light pulse at circadian time 15 (CT15) on day 7 in DD. We distinguished between age groups of 100, 300, 500, 700, and $900 \mathrm{~d}$ and analyzed agerelated changes using SPSS (PASW Statistics 17) by an ANOVA with post hoc Bonferroni-corrected $t$ tests.

\section{Sleep recordings}

Animals, surgery procedures, and data acquisition. Adult male C57BL/6 mice were used in two groups. The first group was recorded at $100 \mathrm{~d}$ of age ( $n=7 ; 24.4 \pm 0.7 \mathrm{~g}$ at the time of surgery). The second group was recorded at $500 \mathrm{~d}(n=5)$ and $700 \mathrm{~d}(n=6 ; 36.9 \pm 0.8 \mathrm{~g}$ at the time of surgery). The animals were kept in a $12 \mathrm{~h} \mathrm{light/dark} \mathrm{cycle} \mathrm{(lights} \mathrm{on} \mathrm{at}$ 8:00 A.M.). Housing conditions were similar to those in behavioral experiments. Under deep anesthesia [ketamine (Aesculaap); xylazine (Bayer); and atropine (Teva Pharmachemie], the animals were implanted with miniature electroencephalogram (EEG) recording screws and electromyogram (EMG) electrodes (Plastics One) as described previously (Deboer et al., 2002, 2007). The EEG and EMG were recorded with a portable system as described previously (Vyazovskiy et al., 2006; Deboer et al., 2007).

Sleep-wake recordings. Mice were individually placed in the recording chambers, connected to the recording setup through a flexible cable and a counterbalanced swivel system. They were allowed to adapt to the experimental condition for at least $3 \mathrm{~d}$. During this period and during the recordings, the animals did not have access to a running wheel. After a $24 \mathrm{~h}$ baseline day, starting at lights on, a $6 \mathrm{~h}$ sleep deprivation was performed, which was followed by $18 \mathrm{~h}$ of recovery. Sleep deprivation was performed by introducing objects (e.g., food, nesting material) into the cage and later by tapping on the cage whenever the animals appeared drowsy or the EEG exhibited slow waves (Deboer et al., 2002). The mice were never disturbed during feeding and drinking.

Data analysis and statistics. Three vigilance states [rapid eye movement (REM) sleep, non-REM (NREM) sleep, and waking] were scored offline in $4 \mathrm{~s}$ epochs by visual inspection of the EEG and EMG signals as well as EEG power density in the slow-wave range as described previously (Deboer et al., 2002). Epochs with artifacts were excluded from the subsequent data analysis of the power spectra, but vigilance states could always be determined. Corresponding light and dark mean values and hourly values of vigilance states and EEG slow-wave activity (SWA) (mean EEG power density between 0.75 and $4.0 \mathrm{~Hz}$ ) in NREM sleep were analyzed.

To test the effect of sleep deprivation and age on light and dark values, paired $t$ tests (sleep deprivation) and Duncan's test (age) were applied. A two-way ANOVA with factors condition and age was applied to test the differences between the groups and experimental conditions. When the ANOVA reached significance, paired and unpaired $t$ tests were applied when appropriate to determine the effects of sleep deprivation and different ages.

In vitro extracellular brain slice recordings.

Animals of 100 or $700 \mathrm{~d}$ were taken from DD. The onset of wheel running activity was determined over at least $7 \mathrm{~d}$ before the experiment to esti- mate the phase of the rhythm. Decapitation and subsequent dissection of the brain was performed at the end of the subjective day (CT12). Preparation of brain slices took place in dim red light and was performed as described previously (VanderLeest et al., 2007, 2009). In the laminar flow slice chamber, the slices (450 $\mu \mathrm{m}$ thick) were stabilized and kept submerged under a thin layer $(\sim 100 \mu \mathrm{m})$ of artificial CSF (ACSF) $\left(36^{\circ} \mathrm{C}\right.$, flow rate $\sim 1.5 \mathrm{ml} / \mathrm{min}$ ) by a tungsten fork and were oxygenated with warmed, humidified $\mathrm{O}_{2}(95 \%)$ and $\mathrm{CO}_{2}(5 \%)$. Slices were left to settle in the chamber for $\sim 1 \mathrm{~h}$ before electrodes were placed and recording was started. Extracellular recording electrodes (insulated 90\% platinum and $10 \%$ iridium, $75 \mu \mathrm{m}$ ) were placed in the left and right SCN to obtain multiunit neuronal activity recordings from both nuclei. In a subset of experiments, we added 4-aminopyridine (4-AP) (5 mM) to the ACSF, perfused for $30 \mathrm{~min}$, and determined the change in electrical activity in percentage of baseline (10 min before the pulse). Amplification and filtering techniques were as described previously (VanderLeest et al., 2007). In parallel, the time of occurrence and the amplitudes of action potentials were digitized and recorded by a data acquisition system (Power1401, Spike2 software; Cambridge Electronics Design) and stored for offline amplitude and subpopulation analysis.

Analysis of multiunit activity. The electrophysiological data were analyzed offline in MATLAB (MathWorks) using custom-made software as described previously (VanderLeest et al., 2007). Multiunit recordings of at least $24 \mathrm{~h}$ were moderately smoothed using a penalized least-squares algorithm (Eilers, 2003).

An analysis of rhythm amplitude was performed in which the total number of action potentials during the electrical activity peak (peak time $\pm 15 \mathrm{~min}$ ) was standardized offline. This allowed for a comparison between young and old slices for multiple sizes of subpopulations (VanderLeest et al., 2009).

Subpopulation analysis. Amplitude data recorded from action potentials were divided into 50 equally sized bins reaching from a low spike threshold level, representing a large number of neurons, to a high threshold including only the activity of a small neuronal subpopulation (Schaap et al., 2003; VanderLeest et al., 2007). In this way, we could describe the circadian activity pattern of small subpopulations of SCN neurons. The timing of the activity of neuronal subpopulations in the SCN was analyzed using MATLAB. The subpopulation activity was smoothed, and the time of maximum firing frequency was analyzed.

Statistical analyses. All values obtained in the multiunit recordings were tested for differences between young and old groups in SPSS using unpaired $t$ tests. Day-night differences in the distribution of subpopulation activity was tested using a $\chi^{2}$ test, and differences between groups and timing were tested using a $\chi^{2}$ test in a cross tabulation with daynight and young-old as groups. $p$ values $<0.05$ were considered to be significantly different. Additionally, curve-fitting procedures were performed in Igor Pro, using Multi-Peak Fit version 2.05. Curve fitting results were judged by Akaike's information criterion (AIC). The bestfitting model is indicated by the lowest AIC value.

\section{Whole-cell patch-clamp recording}

Brain slice preparation. A group of 19 old mice ( $700 \mathrm{~d}$; day, $n=10$; night, $n=9$ ) and 23 young mice ( $100 \mathrm{~d}$; day, $n=10$; night, $n=13$ ) were used for patch-clamp recording. Animals were killed within $1 \mathrm{~h}$ after lights on [Zeitgeber time 0 (ZT0) to ZT1] for recording during the day (ZT3-ZT7) and at the end of the day (ZT11-ZT12) for recording during the night (ZT15-ZT19). Brains were removed after decapitation, and coronal hypothalamic slices ( $250 \mu \mathrm{m}$ thick) containing the SCN were cut in cold oxygenated ACSF with elevated $\mathrm{Mg}^{2+}(4 \mathrm{~mm})$ and reduced $\mathrm{Ca}^{2+}(1 \mathrm{~mm})$ using a vibrating microtome (VT 1000S; Leica). Brain slices were then transferred to normal ACSF ( $\mathrm{pH} 7.2-7.4$ ) containing (in $\mathrm{mM}$ ) 116.4 $\mathrm{NaCl}, 5.4 \mathrm{KCl}, 1 \mathrm{NaH}_{2} \mathrm{PO}_{4}, 0.8 \mathrm{MgSO}_{4}, 1.8 \mathrm{CaCl}_{2}, 23.8 \mathrm{NaHCO}_{3}$, and 15.1 glucose (Merck), warmed up to $36^{\circ} \mathrm{C}$ for $20-30 \mathrm{~min}$, and then remained at room temperature for at least $1 \mathrm{~h}$ before recordings.

Patch-clamp recordings. For patch-clamp recordings, slices were placed in a recording chamber (RC-26 G; Warner Instruments) and secured with a slice hold-down. Pipettes (5-7 M $\Omega$ ) were fabricated from borosilicate glass (WPI B150F-4) using a commercial electrode puller (PC-10 Narishige). The solution in the patch pipette contained the following (in 

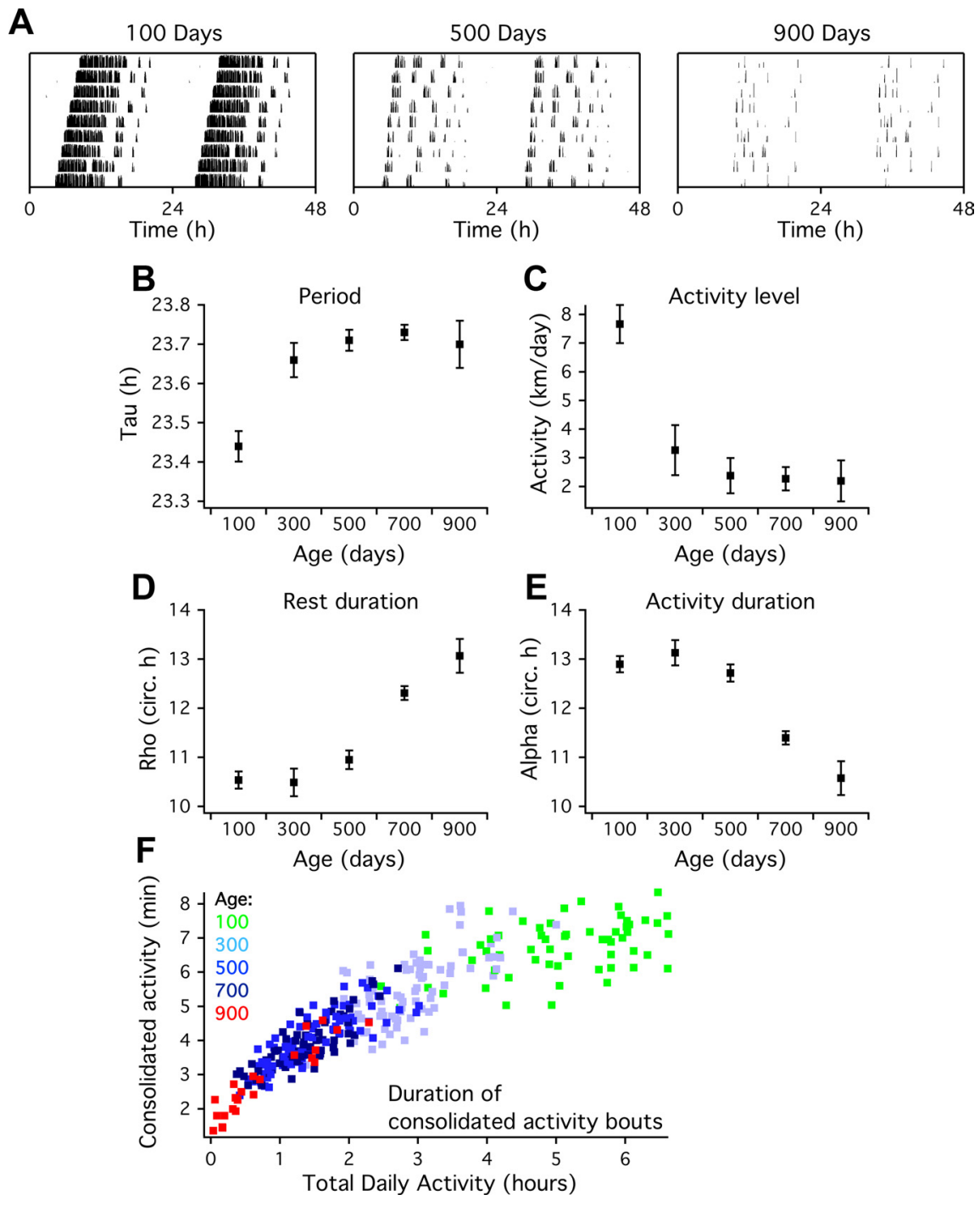

Figure 1. Age-related changes in the circadian pattern of behavioral activity. $A$, Representative plots showing $9 \mathrm{~d}$ of wheelrunning activity in DD in young ( $100 \mathrm{~d}$ ), middle-aged (500 d), and old ( $900 \mathrm{~d}$ ) mice. Data are double plotted for clarity. $\boldsymbol{B}$, The cycle length in DD $(\tau)$ shows a marked increase between 100 and $300 \mathrm{~d}$ old and remains constant at later ages. $C$, The amount of daily activity decreases between 100 and $300 \mathrm{~d}$. D. The duration of the resting phase $(\rho)$ is constant in the first three age groups and increases at $700 \mathrm{~d}$. $\boldsymbol{E}$, The duration of the active phase $(\alpha)$ decreases at $700 \mathrm{~d}$. The duration of $\alpha$ and $\rho$ are plotted as a fraction of the circadian cycle $(\tau / 24)$ to correct for the age-related period lengthening. $F$, Longitudinal analysis of individual running wheel records ( $n=5$ animals; colored markers represent the average activity bout duration during $7 \mathrm{~d}$ ) shows that both the duration of consolidated activity bouts and time spent in the wheel declines as a function of age. All parameters in $\boldsymbol{B}-\boldsymbol{E}$ show significant changes with age.

mM): 112.5 K-gluconate, 1 BAPTA, 10 HEPES, 5 MgATP, 1 GTP, 0.1 leupeptin, 10 phosphocreatine, $4 \mathrm{NaCl}, 17.5 \mathrm{KCl}, 0.5 \mathrm{CaCl}_{2}$, and $1 \mathrm{MgCl}_{2}$ (Sigma-Aldrich). The $\mathrm{pH}$ was adjusted to 7.25-7.3, and the osmolality was adjusted to $290-300 \mathrm{mOsm}$. Recordings were performed in the acute slice preparation [similar to the multiunit activity (MUA) recordings] using a patch-clamp amplifier (EPC 10-2; HEKA) performing capacitance compensation and monitoring series resistance $\left(R_{\text {series }}\right)$. Recordings with $R_{\text {series }}$ higher than $40 \mathrm{M} \Omega$ or with holding currents $\left(I_{\text {hold }}\right)$ larger than $100 \mathrm{pA}$ (at $V_{\text {hold }}=-70 \mathrm{mV}$ ) were excluded from additional analysis.

After seal formation, cell-attached recordings were first performed in normal ACSF to measure the spontaneous firing rate (SFR) of cells. Postsynaptic currents were recorded at holding potential of $-70 \mathrm{mV}$ after breaking the membrane and gaining electrical access to the cell. With the chosen filling solution, the reversal potential for IPSCs is between -40 and $-50 \mathrm{mV}$ (Itri et al., 2004). Subsequently, GABAmediated IPSCs appear as inward currents because the reversal potential for chloride was more positive than the holding potential. Synaptic events were excluded if the decay time was $<4.6 \mathrm{~ms}$ to eliminate AMPAmediated events (Michel et al., 2002).

Characterization of DR and A-type $K^{+}$currents. Recording and isolation of $\mathrm{K}^{+}$currents were performed as described previously (Itri et al., 2005, 2010). To isolate delayed-rectifier (DR) potassium currents, a voltage protocol was applied consisting of $100 \mathrm{~ms}$ prepulse at $-100 \mathrm{mV}$, followed by $400 \mathrm{~ms}$ steps at a progressively depolarized potential that was composed of 12 sweeps from -50 to $+60 \mathrm{mV}$. A 20 $\mathrm{ms}$ pause at $-100 \mathrm{mV}$ was applied to bring channels back to activation mode. For recording DR currents, first a control solution containing bicuculine (20 $\mu \mathrm{M}$; Sigma-Aldrich), cadmium (25 $\mu \mathrm{m}$; Sigma-Aldrich), and tetrodotoxin $(0.5 \mu \mathrm{M}$; Tocris Bioscience) was applied to block $\mathrm{GABA}_{\mathrm{A}}$ receptors, voltage-activated calcium channels, and fast voltage-gated sodium channels; respectively. Subsequently, tetraethylammonium (TEA) (1 and $20 \mathrm{~mm}$; SigmaAldrich) was added to the bath solution to block fast (FDR) and slow (SDR) DR K ${ }^{+}$channels, respectively. The DR voltage protocol was applied after 3-7 min of each drug treatment. In the analysis, the traces in 1 mM TEA were subtracted from traces in control solution to achieve isolated FDR currents $\left(I_{\text {control }}-I_{1 \mathrm{~mm}}\right.$ TEA). In addition, traces recorded in the higher concentration of TEA were subtracted from traces of lower concentration of TEA $\left(I_{1 \mathrm{~mm}}\right.$ TEA $-I_{20 \text { mм }}$ TEA $)$ to isolate SDR currents.

A-type potassium currents $\left(I_{\mathrm{A}}\right)$ were isolated using voltage steps from two different holding potentials. Voltage steps $(150 \mathrm{~ms},-60$ to +60 $\mathrm{mV}, 10 \mathrm{mV}$ steps) were applied from either $-90 \mathrm{mV}$ for maximal activation or after inactivation of $I_{\mathrm{A}}$ at $-45 \mathrm{mV}$. To isolate $I_{\mathrm{A}}$ current, traces of the inactivation protocol were subtracted from traces of the activation protocol $\left(I_{\mathrm{IA}-90}-\mathrm{I}_{\mathrm{IA}-45}\right)$. To isolate the $I_{\mathrm{A}}$ from FDR, $I_{\mathrm{A}}$ was measured after blocking FDR with $1 \mathrm{~mm}$ TEA. Importantly, the amplitude of the $I_{\mathrm{A}}$ current was similar before and after blockage of the FDR.

Leak subtraction was performed during all voltage protocols using four subpulses with one-quarter of the test pulse amplitude and reversed polarity given from a holding potential of $-80 \mathrm{mV}$.

Statistical analyses. Unpaired $t$ tests in SPSS were applied to test age-related differences. All means are reported \pm SEM in both the text and figures. The differences in the proportions of silent and active cells or regular and irregular cells were determined with $\chi^{2}$ test.

\section{Results}

\section{Behavioral activity recordings}

Behavioral recordings were performed in mice of 100, 300, 500, 700 , and $900 \mathrm{~d}$ of age (Fig. 1). In all behavioral circadian parameters, significant changes were observed in the course of aging, but noticeably these changes occurred at different stages. The circadian period $(\tau)$ for animals of $300 \mathrm{~d}$ was significantly longer compared with animals of $100 \mathrm{~d}$ ( $p<0.01$; Fig. $1 B$, Table 1$)$. A $42 \%$ decrease in the total amount of activity also occurred at this age $(p<0.01$; Fig. $1 C)$. The time course of the changes in activity 
Table 1. Age-related alterations in the freerunning period $(\tau)$, daily activity, resting duration $(\rho)$, activity duration $(\alpha)$, intradaily variability (IV), periodogram peak amplitude, and the light induced phase delay (CT15). Data represent mean \pm SEM

\begin{tabular}{|c|c|c|c|c|c|c|c|c|c|c|c|}
\hline Group & Light & 1 & $n$ & 2 & $n$ & 3 & $n$ & 4 & $n$ & 5 & $\bar{n}$ \\
\hline Age [days] & & 100 & & 300 & & 500 & & 700 & & 900 & \\
\hline$\tau[\mathrm{h}]$ & DD & $23.44 \pm 0.04$ & 77 & $23.66 \pm 0.04$ & 19 & $23.71 \pm 0.03$ & 36 & $23.73 \pm 0.02$ & 70 & $23.70 \pm 0.06$ & 10 \\
\hline Activity $[\mathrm{km}]$ & DD & $7.67 \pm 0.67$ & 52 & $3.27 \pm 0.88$ & 16 & $2.38 \pm 0.62$ & 29 & $2.27 \pm 0.40$ & 66 & $2.20 \pm 0.72$ & 10 \\
\hline$\rho[$ circh $]$ & DD & $10.78 \pm 0.18$ & 72 & $10.80 \pm 0.23$ & 16 & $11.00 \pm 0.20$ & 29 & $12.43 \pm 0.14$ & 66 & $13.27 \pm 0.35$ & 10 \\
\hline$\alpha[\operatorname{circh}]$ & $\mathrm{DD}$ & $13.22 \pm 0.18$ & 72 & $13.20 \pm 0.23$ & 16 & $13.0 \pm 0.20$ & 29 & $11.57 \pm 0.14$ & 66 & $10.74 \pm 0.35$ & 10 \\
\hline IV & LD & $0.36 \pm 0.05$ & 10 & $0.51 \pm 0.05$ & 10 & $0.53 \pm 0.04$ & 10 & $0.71 \pm 0.05$ & 10 & $0.99 \pm 0.15$ & 10 \\
\hline Periodogram peak & LD & $0.44 \pm 0.02$ & 10 & $0.23 \pm 0.03$ & 10 & $0.16 \pm 0.02$ & 10 & $0.11 \pm 0.02$ & 10 & $0.10 \pm 0.04$ & 10 \\
\hline Phase delay $[\mathrm{h}]$ & DD & $-2.00 \pm 0.11$ & 20 & & & & & $-1.66 \pm 0.10$ & 31 & & \\
\hline
\end{tabular}

levels and $\tau$ were similar, and both showed no additional changes with increasing age. The duration of the resting period $(\rho)$ was stable for almost 2 years but increased significantly at $700 \mathrm{~d}$ (Fig. $1 D)$. Concomitant, the duration of the active period $(\alpha)$ decreased in this age group (Fig. $1 E$ ). The increments in $\rho$ appeared progressive, and some animals lost their circadian rhythm almost completely at the last stage of their life.

As the aging process progressed, the distribution of behavioral activity over the light/dark cycle changed. Young animals showed a characteristic period of several hours of intense, consolidated locomotor activity in the first half of the night that disappeared in most animals before the age of $500 \mathrm{~d}$. The duration of consolidated running bouts decreased from $6.8 \pm 0.4 \mathrm{~min}$ in animals of $100 \mathrm{~d}$ to $2.8 \pm 0.6 \mathrm{~min}$ in animals of $700 \mathrm{~d}$ (Fig. 1 F). Periodogram analysis showed a decrease in the rhythm strength (Table 1). Fragmentation of the activity rhythm, as assessed by calculating the IV, revealed a progressive increase with age (Table 1). Old mice (700 d, $n=31$ ) showed a significantly smaller phaseshifting capacity in response to a light pulse compared with young animals ( $100 \mathrm{~d}, n=20$; Table 1 ).

\section{Sleep-wake recordings}

The changes in circadian and ultradian activity patterns were reflected in the sleep-wake changes in the course of aging. The groups of 500 and $700 \mathrm{~d}$ showed less waking and more NREM sleep compared with the $100 \mathrm{~d}$ group, particularly during the dark period (Fig. 2). The decrease in waking in the dark period was mainly attributable to a substantial decrease in waking episode duration.

The $700 \mathrm{~d}$ group had a shorter period of increased consolidated waking compared with the younger groups (Fig. 2). This was mainly attributable to an earlier offset of increased waking in the course of the dark period, which was significantly different between the 100 and $700 \mathrm{~d}$ groups. No differences were found in SWA in response to sleep deprivation between the age groups (data not shown). The findings indicate clear changes in circadian modulation but only minor changes in sleep homeostatic mechanisms in the course of aging.

\section{In vitro MUA recordings}

Because the aging phenotype was fully present at $700 \mathrm{~d}$, all electrophysiological recordings were performed in this age group. Recordings of extracellular MUA in slices from old animals (700 d) revealed an earlier peak in firing rate rhythm (CT $5.40 \pm$ $0.27 \mathrm{~h}, n=29)$ compared with young ( $100 \mathrm{~d})$ mice $(6.39 \pm 0.29 \mathrm{~h}$, $n=22, p<0.05$; Fig. $3 A, B)$. The MUA rhythm also showed an advanced ascending slope in old mice (time of half-maximum: old CT, $0.45 \pm 0.33 \mathrm{~h}, n=29$; young CT, $2.00 \pm 0.28 \mathrm{~h}, n=22$; $p<0.001$ ), and a reduction in the steepness of the ascending slope of the MUA rhythm was found in old compared with young
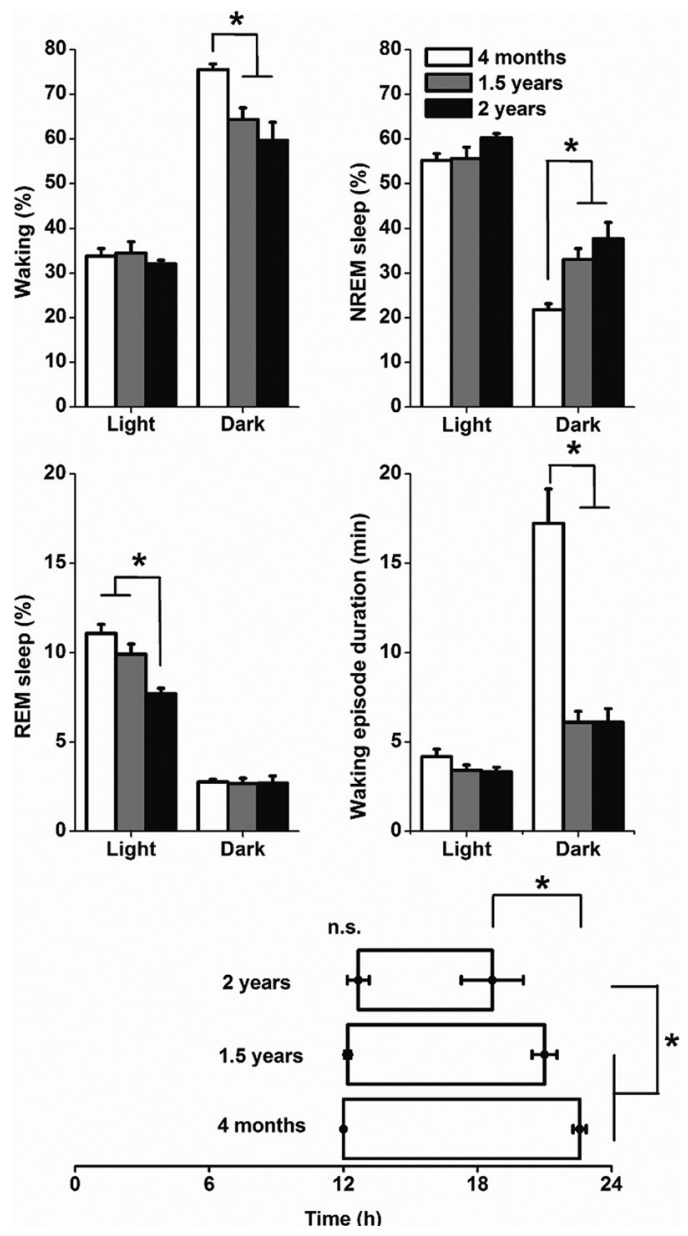

Figure 2. Top, Middle, Effect of aging on vigilance states and waking episode duration in the light and dark period of animals of $100 \mathrm{~d}(n=7), 500 \mathrm{~d}(n=5)$, and $700 \mathrm{~d}(n=6)$. Vigilance state values are expressed as mean \pm SEM percentage of $12 \mathrm{~h}$ recording time, waking episode duration is expressed in minutes \pm SEM. The light and dark values were significantly different for all vigilance states and for waking episode duration $(p<0.05)$. Bottom, Distribution of waking during the day. Indicated are the nighttime hours the animals spend on average $>70 \%$ awake. Note the equal onset of increased waking at lights off (time $12 \mathrm{~h}$ ) but the unequal ending of waking at the end of the night. Asterisks indicate differences in the duration and offset time of increased waking; ${ }^{*} p<0.05$.

mice (Fig. 3C). An offline analysis showed that the amplitude of the electrical rhythm was significantly lower in old mice (Fig. $3 D$ ). The decrease in rhythm amplitude is explained by a higher trough level in old animals for all population sizes, with this difference being stronger when larger population sizes were considered (Fig. $3 D, E$ ).

Subpopulations were recorded to determine the phase distribution of neuronal activity patterns in young and old animals. In 

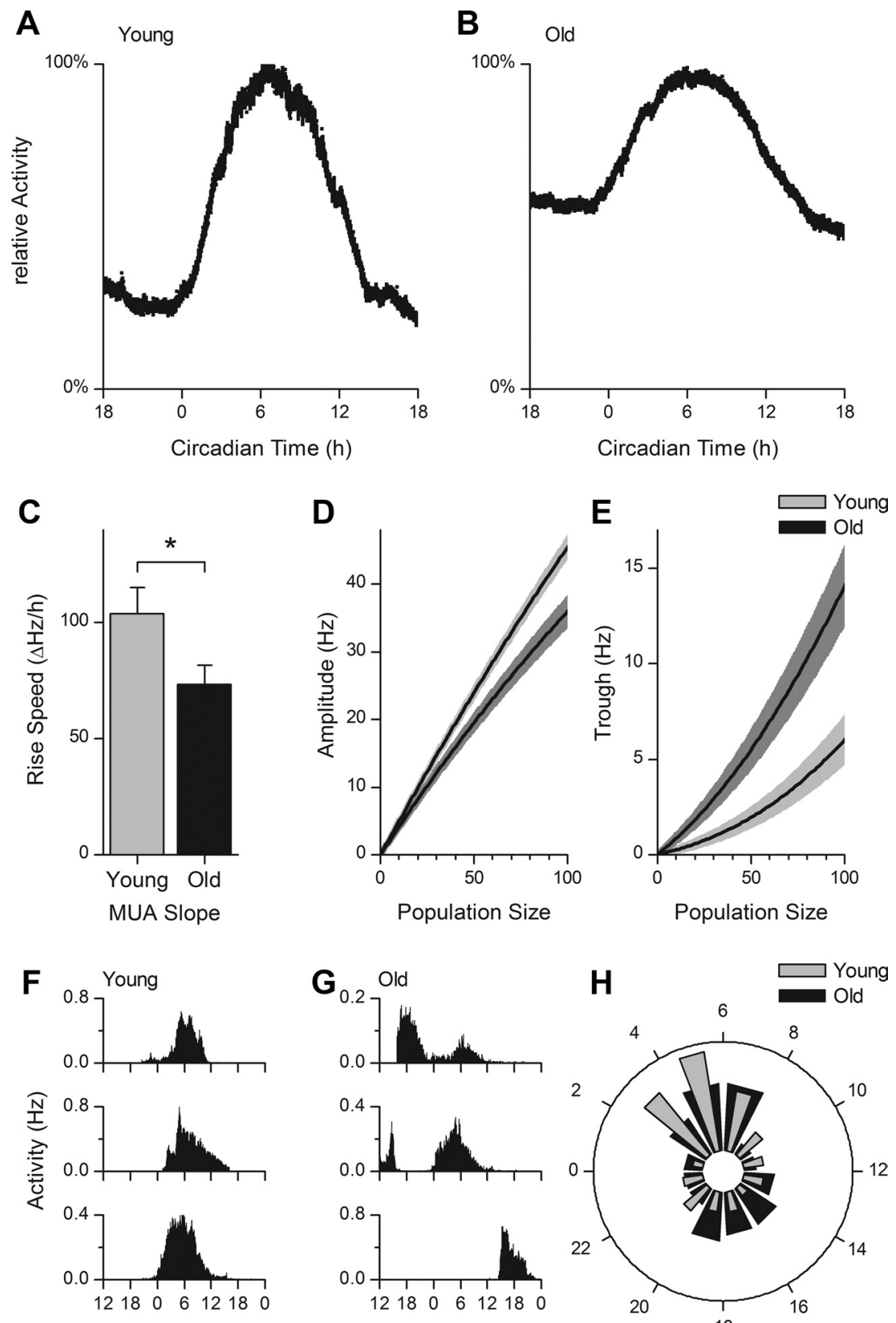

Circadian Time (h)

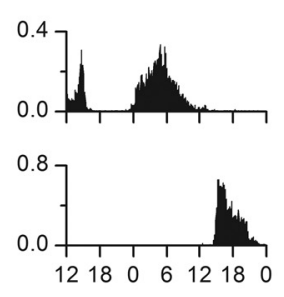

Circadian Time (h)

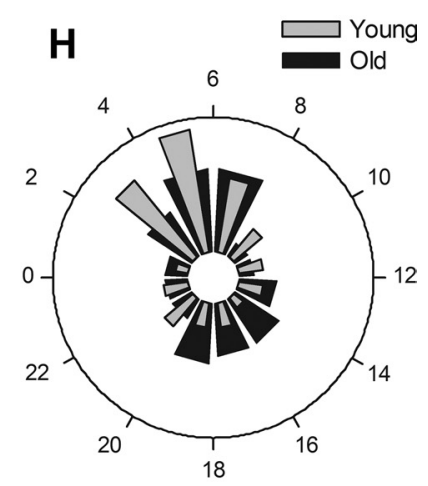

Circadian Time $(\mathrm{h})$

Figure 3. Extracellular recordings of electrical activity in brain slices from young and old animals. $A, B$, Examples of normalized MUA patterns as a function of CT. In slices from old animals, a higher trough level is observed. $C$, The rising slope of the multiunit electrical discharge pattern is reduced in old animals (old, $73.21 \pm 8.29 \Delta \mathrm{Hz} / \mathrm{h}$; young, $104.02 \pm 10.97 \Delta \mathrm{Hz} / \mathrm{h} ;{ }^{*} p<0.05$ ). , Average \pm SEM rhythm amplitude as a function of population size. The difference in trough to peak amplitude increases with increasing population size (at level 100: old, $35.95 \pm 1.84 \mathrm{~Hz}$; young, $45.43 \pm 1.12 \mathrm{~Hz} ; p<0.01$ ). $\boldsymbol{E}$, The lower amplitude in slices from old animals is directly attributable to an increase in the trough in old animals (at level 100: old, $14.08 \pm 1.81 \mathrm{~Hz}$; young, $6.01 \pm 1.10 \mathrm{~Hz} ; p<0.01) . \boldsymbol{F}, \boldsymbol{G}$, Raw subpopulation activity (in hertz) profiles, recorded from young $(\boldsymbol{F})$ and old $(\boldsymbol{G})$ animals, as a function of $\mathrm{CT}$ (hours). Multiple subpopulation peak times were observed in old mice. In young mice, most subpopulations had an activity peak during projected midday (CT6). $\boldsymbol{H}$, The time of maximal activity of small neuronal subpopulations are displayed in a phase histogram, with the midday corresponding with CT6. In young animals, there is a significant difference in the number of subpopulation peaks in the day versus night $(p<0.01)$. In old animals, however, a large number of subpopulations have their peak at night, and there is no significant difference in the number of active populations during the day and night $(p=0.72)$.

young animals, the distribution of subpopulation activity displayed a unimodal pattern with a maximum during midday. In old animals, a secondary peak was observed in anti-phase to the main peak, causing significant differences in the distribution of subpopulation activity between old and young slices (Fig. $3 \mathrm{~F}-\mathrm{H}$, $p<0.05)$.
The timing of subpopulation activity in old animals was best described by fitting two Gaussian distributions over the $24 \mathrm{~h}$ cycle (mid of peak 1, CT $5.5 \pm 0.3 \mathrm{~h}$; mid of peak 2 , CT $17.0 \pm 0.6 \mathrm{~h}$; $\mathrm{AIC}=47$ for bimodal distribution; AIC $=57$ for unimodal distribution), whereas in young animals it was best described by a single Gaussian distribution (peak time, CT $4.8 \pm 0.2 \mathrm{~h}$; AIC $=45$ for unimodal distribution)

\section{Patch-clamp recordings}

Membrane properties and excitability

To investigate whether the observed changes in the network may be caused by changes in membrane properties of individual neurons, patch-clamp recordings were performed. The results revealed striking differences in membrane properties in old versus young mice. The resting membrane potential $\left(V_{\mathrm{m}}\right)$ of SCN neurons from old mice was significantly more depolarized during the night compared with $V_{\mathrm{m}}$ in young mice (young, $-48.53 \pm$ $1.38 \mathrm{mV}$; old, $-41.04 \pm 1.1 \mathrm{mV} ; p<$ 0.001 ; Fig. $4 A, B)$. This resulted in a lack of circadian modulation in membrane potential in old mice, whereas in young mice, $V_{\mathrm{m}}$ was significantly more depolarized during the day $(-41.43 \pm 0.84 \mathrm{mV})$ than during the night $(p<0.01)$. Input resistance $\left(R_{\text {input }}\right)$, as calculated from the values of $I_{\text {hold }}$, $V_{\text {hold }}$, and $R_{\text {access }}$, was significantly higher during the night in old (1.24 $\pm 0.1 \mathrm{G} \Omega$ ) compared with young $(0.76 \pm 0.04 \mathrm{G} \Omega ; p<0.0001)$ neurons. A daily rhythm of $R_{\text {input }}$ was observed in young but not in old cells, with higher $R_{\text {input }}$ during the day $(1.12 \pm 0.06 \mathrm{G} \Omega$; $p<0.01$ ) compared with the night (Fig. $4 C)$. During the day, SFR in old cells was significantly lower compared with young neurons $(43 \%)$. The day-night difference in SFR observed in young mice was not present in the old mice (Fig. $4 D, E$ ). We calculated the coefficient of variation of the interspike interval to determine the firing pattern of the SCN cells. No significant differences were found between day and night or between the two age groups. There was a trend to more silent cells during the day and less during the night (day, $31 \%$ in old vs $19 \%$ in control; night, $37 \%$ in old vs $21 \%$ in controls; $p>$ $0.05)$. This trend is consistent with findings of Nygård et al. (2005). Depolarized membrane states have been shown recently to silence SCN neurons in the middle of the day in per1expressing cells (Belle et al., 2009), but, in our data, the mean $V_{\mathrm{m}}$ of the silent cells did not differ from mean $V_{\mathrm{m}}$ of active cells in any of the groups. Cell capacitance of SCN neurons was significantly reduced in old compared with young animals (Fig. $4 F$ ). 
To investigate age-dependent changes in synaptic properties, we recorded spontaneous postsynaptic currents in wholecell recordings at a holding potential of $-70 \mathrm{mV}$. Most of the synaptic events were inhibitory (IPSCs) and were blocked by application of the $\mathrm{GABA}_{\mathrm{A}}$ receptor antagonist bicuculine ( $20 \mu \mathrm{M}$; data not shown). We did not find significant differences in the mean frequency of IPSCs between old and young cells (day, $p=0.36$; night, $p=$ 0.13 ) nor between day and night measurements (old, $p=0.12$; young, $p=0.39$ ). The amplitude of IPSCs was significantly smaller in old mice with no modulation between day and night as observed in the IPSC amplitude of young mice (Fig. 5).

Age-related changes in $\mathrm{K}^{+}$currents To identify possible mechanisms for the lack of circadian spike frequency modulation in old SCN neurons, we recorded FDR, SDR, and $I_{\mathrm{A}} \mathrm{K}^{+}$currents. FDR currents from young mice were significantly larger during the day compared with the night $(p<0.05$; Fig. $6 A, C)$, confirming previous findings (Itri et al., 2005). In contrast, FDR currents in SCN neurons from old mice did not differ between the day and the night ( $p=0.6$; Fig. $6 B)$. FDR currents recorded during the night were significantly larger in old mice compared with young $(p<0.01$; Fig. $6 C)$. During the day, the same tendency was observed. SDR currents did not show significant differences between the two age groups (Fig. 6G-I).

Consistent with previous work (Itri et al., 2010), the $I_{\mathrm{A}}$ current amplitude in $\mathrm{SCN}$ neurons from young mice was larger during the day compared with the night $(p<0.05$; Fig. $6 D, F)$. The amplitude of $I_{\mathrm{A}}$ currents during the day was significantly reduced in old compared with young neurons at $+60 \mathrm{mV}(p<0.01$; Fig. $6 F$ ). Additional analysis of the activation curves for $I_{\mathrm{A}}$ revealed significant differences over a voltage range between +10 and +60 $\mathrm{mV}(p<0.05)$. No day-night difference in the $I_{\mathrm{A}}$ amplitude was present in old animals (Fig. 6E).

To functionally test whether modulation of $I_{\mathrm{A}}$ current could explain agedependent changes in SFR modulation, we applied 4-AP at a concentration shown previously to block $I_{\mathrm{A}}$ (5 mM; Itri et al., 2010) to slices from young animals and recorded MUA in the SCN. Application during the day significantly reduced SFR by $21.6 \pm 1.9 \%(n=13$ traces; $p<0.0001)$.

\section{Discussion}

Our data constitute unprecedented evidence for cellular membrane deficits in circadian pacemaker neurons of old mice. The severity of these effects was surprising in view of the current hypothesis that aging affects predominantly the brain network $0.01,{ }^{* * *} p<0.001$.
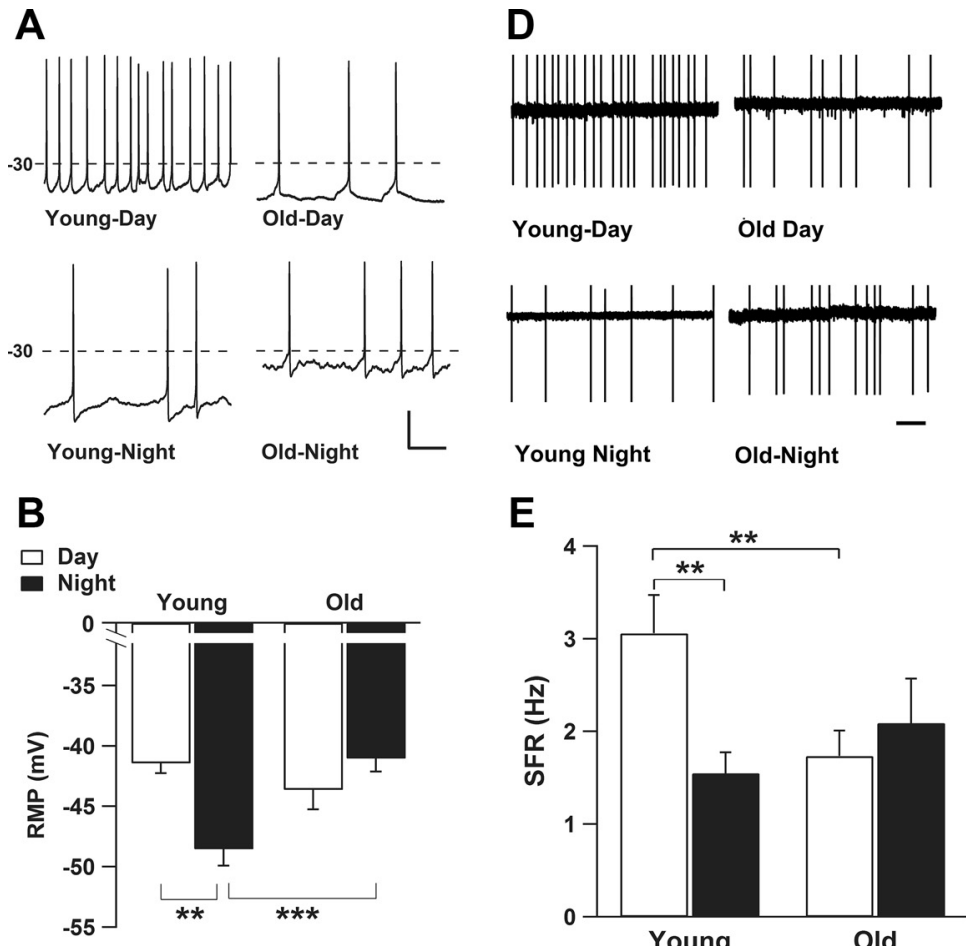

E

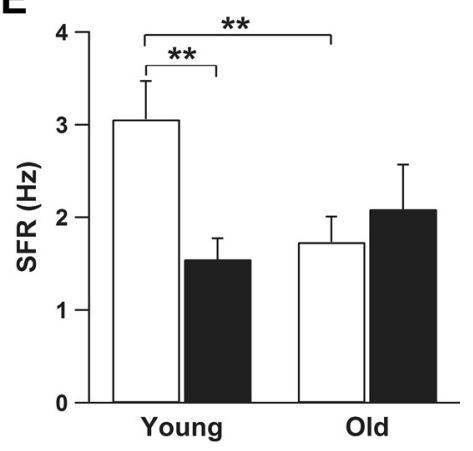

$\mathbf{F}$

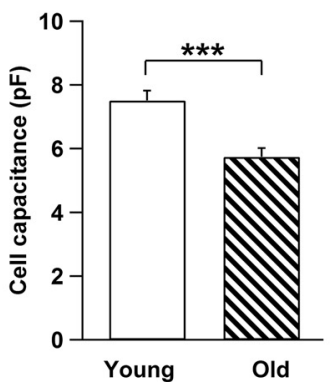

Figure 4. Active and passive membrane properties in young and old SCN neurons. $\boldsymbol{A}$, Examples of whole-cell current-clamp recording of membrane potential of SCN neurons. Recordings from young animals showed larger differences in resting membrane potential (RMP) between day and night compared with old animals. Calibration: $20 \mathrm{mV}, 200 \mathrm{~ms}$. B, Bar graph depicts that mean \pm SEM resting membrane potential of young neurons is more depolarized during the day $(n=14)$ compared with the night $(n=32)$. Resting membrane potential from old animals was more depolarized during the night $(n=25)$ with no significant difference compared with day values $(n=24 ; p=0.192)$. $C, R_{\text {input }}$ is decreased during the night in young SCN neurons $(n=41)$ compared with cells recording during the day $(n=30)$. Old SCN neurons indicated a higher $R_{\text {input }}$ during the night $(n=25)$ compared with young cells but with no difference to the mean $R_{\text {input }}$ of aged cells recorded during the day $(n=26 ; p=0.063)$. D, Examples of SFR measured with cell-attached extracellular recording methods demonstrate a rhythm in firing frequency in young neurons that was absent in recordings from old mice. Scale bar, 1 s. E, Bar graph of mean \pm SEM SFR reveals a significant higher rate during the day of young SCN neurons $(n=25)$ compared with the night $(n=35)$. In old neurons, SFR is significantly reduced during the day $(n=$ 29). Old neurons show no difference in mean SFR between the day and night ( $n=19 ; p=0.492)$. $\boldsymbol{F}$, The cell capacitance, which reflects the cell membrane area, was significantly less in aged neurons $(n=53)$ compared with young group $(n=72)$. ${ }^{* *} p<$

level. Longitudinal behavioral and sleep studies showed an agerelated decrease in the total activity levels and a change in the distribution of activity, rest, and sleep over the circadian cycle but no changes in homeostatic sleep regulation, suggesting an important role for the SCN in age-related sleep disorders. Electrophysiological recordings showed changes in neuronal phase distribution that can explain the decrease in circadian amplitude in aged animals. Patch-clamp recordings revealed specific alterations in membrane function and in GABAergic activity during the day and night. We will discuss the findings from the cellular toward the behavioral level and will evaluate how the cellularand network-level changes could lead to the aged phenotype. 
A

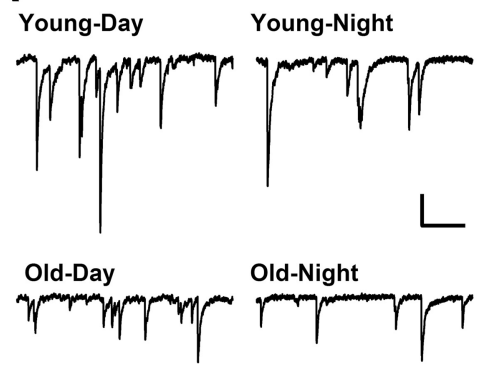

B



Figure 5. GABAergic IPSCs recorded from young and old SCN neurons. $\boldsymbol{A}$, Examples of IPSCs from young and old neurons. Note the reduced amplitude of IPSC in old SCN neurons. Calibration: 20 pA, $200 \mathrm{~ms}$. $\boldsymbol{B}$, The amplitude of IPSCs shows a significant rhythm between day $(n=21)$ and night $(n=36)$ in young neurons $(p<0.05)$. In old neurons, the rhythm of the IPSC amplitude is diminished between day $(n=28)$ and night $(n=23)(p=0.117)$. Each bar represents mean \pm SEM. ${ }^{*} p<0.05,{ }^{* *} p<0.0001$.

A

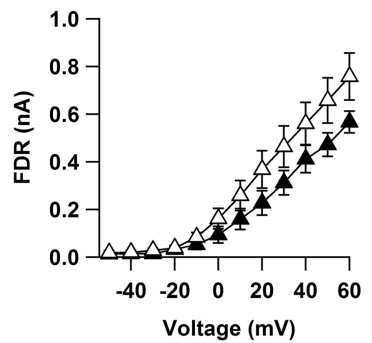

B

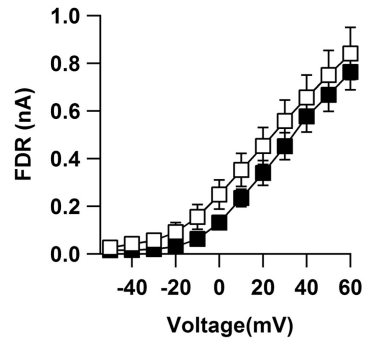

C

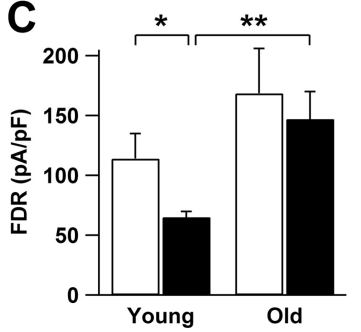

D

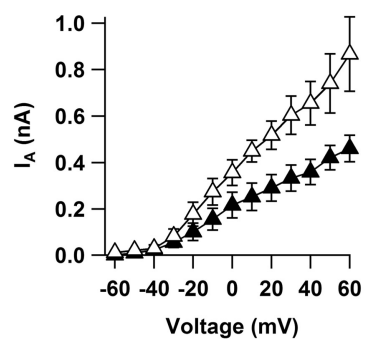

E

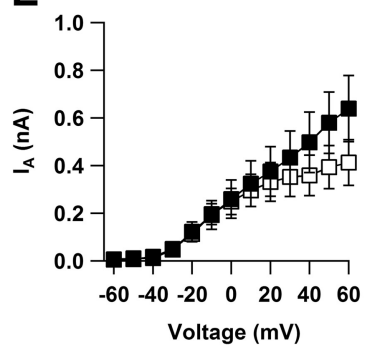

$\mathbf{F}$

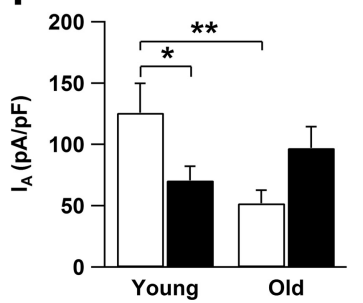

G



H

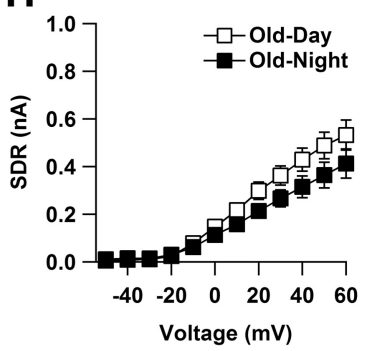

I



Figure 6. Age-dependent changes in FDR and $I_{\mathrm{A}} \mathrm{K}^{+}$current amplitude but not in SDR currents. $\boldsymbol{A}, \boldsymbol{D}, \mathrm{FDR}$ and $I_{\mathrm{A}}$ current amplitude indicated rhythmicity in young SCN cells. $\boldsymbol{B}, \boldsymbol{E}$, In the aged SCN cells, both currents lost their circadian rhythm. $\boldsymbol{C}$, Mean normalized FDR current amplitude (at $+60 \mathrm{mV}$ ) in young SCN cells was significantly greater during the day $(n=11)$ than the night ( $n=11)$. In aged SCN neurons, FDR current amplitude did not show a significant difference between the day $(n=12)$ and the night $(n=13)$. FDR currents recorded during the night in aged cells were significantly larger than those recorded in young SCN cells. $\boldsymbol{F}$, Mean normalized $I_{\mathrm{A}}$ current amplitude (at $+60 \mathrm{mV}$ ) in young neurons was larger during the day $(n=5)$ than during the night $(n=13)$. In old animals, there was no difference in $I_{A}$ current amplitude between the day $(n=9)$ and the night $(n=12)$. $I_{A}$ current amplitude was significantly lower during the day in old compared with young neurons. G, SDR currents did not vary between day $(n=9)$ and night $(n=12)$ in young cells $(p=0.39)$. $\boldsymbol{H}$, These currents did not also vary between day $(n=9)$ and night $(n=7)$ in old cells $(p=0.21)$. $I$, There was no significant difference between the values recorded from old and young groups, during the day $(p=0.36)$ or night $(p=0.14) .{ }^{*} p<0.05,{ }^{* *} p<0.01$.

\section{Membrane properties}

Patch recordings from SCN neurons of 2-year-old mice revealed a substantial loss of circadian clock function at the cellular level. The recordings showed that aged SCN neurons have a signifi- cantly more depolarized resting membrane potential at night compared with young animals and lack a circadian modulation of membrane potential, which is a hallmark feature of most SCN neurons (Brown and Piggins, 2007). Membrane potential may be regulated by a TEAsensitive $\mathrm{K}^{+}$channel (Kuhlman and McMahon, 2004). In our study, the aged SCN neurons showed a decrease in membrane conductance during the night, suggesting an impairment of $\mathrm{K}^{+}$channel regulation.

We confirmed a rhythm in the amplitude of the FDR current in SCN neurons of young mice and showed that this rhythm was absent in old mice. Because the FDR current plays an important role in the circadian modulation of firing rate in SCN neurons (Itri et al., 2005), this absence may account for the lack of the rhythm in SFR in old neurons. However, the major differences in FDR currents were observed during the night, suggesting that additional mechanisms are involved. We recorded $I_{\mathrm{A}}$ currents in young animals and confirmed the presence of a circadian rhythm that peaks during the day (Itri et al., 2010). In old animals, we found lowered amplitude of the $I_{\mathrm{A}}$ during the day, leading to a blunted circadian rhythm. Although activation of $I_{\mathrm{A}}$ could have differential effects on SFR, blockage during the day led to a decrease in SFR rendering the aged phenotype. Thus, the daytime decrease in $I_{\mathrm{A}}$ may contribute to the daytime reduction in SFR in aged cells. Importantly, aging had no impact on the amplitude of SDR currents that were recorded from the same cells, indicating that age-related changes in $I_{\mathrm{A}}$ and FDR currents are specific alterations that occur with aging.

The enhancement of FDR currents during the night in aged SCN neurons is in agreement with changes in action potential waveform observed in other areas of the aging brain like in layer $\mathrm{V}$ pyramidal neurons in the monkey prefrontal cortex. Similar to our nighttime recordings, firing rate was unchanged, and a role for the DR $\mathrm{K}^{+}$current was proposed (Luebke and Chang, 2007). Mechanisms of age-related changes in ion channels may be manifold and include altered gene expression (Erraji-Benchekroun et al., 2005) and increased oxidative stress. The latter affects different cation and $\mathrm{K}^{+}$channels, such as DR and $I_{\mathrm{A}}$ currents (Cai and Sesti, 2009). These age-dependent changes in ion conductances not only affect the cell excitability and the magnitude of $I_{\mathrm{A}}$ and DR currents, but importantly, they also lead to changes in neuronal communication involved in synchronization within the SCN. 


\section{Desynchronized neuronal activity patterns}

We observed a $25 \%$ decrease in the amplitude of electrical activity rhythms in old mice, which was mainly caused by an elevation of the nighttime activity of SCN neurons. The reduction in amplitude corresponds with previous findings in SCN slices (Satinoff et al., 1993; Watanabe et al., 1995) and with recent in vivo recordings in the SCN of aged animals (Nakamura et al., 2011). Our present data show that a change in circadian phase coherence may underlie the amplitude reduction. In young animals, $71 \%$ of the neuronal subpopulations were active during the subjective day, whereas in old mice, this dropped to $52 \%$. Remarkably, a significant number of subpopulations appeared active during the night and clustered around CT18. This is in anti-phase to the main activity peak and resulted in a bimodal distribution of activity patterns.

Both VIP and GABA are neurotransmitters that play a role in coupling and have been reported to decrease in the course of aging (Chee et al., 1988; Liu and Reppert, 2000; Albus et al., 2005; Aton et al., 2005; Nygård and Palomba, 2006; Duncan et al., 2001). In young animals, we observed a circadian modulation of GABAergic IPSC amplitude that was absent in old animals. Moreover, the mean amplitude of IPSCs was considerably reduced in old SCN neurons, which could be related to the $30 \%$ reduction in cell capacitance (an equivalent to membrane surface) that we found in old SCN neurons. The shortening of dendrites and loss of spines in old SCN neurons, as well as the dendritic location of $\mathrm{GABA}_{\mathrm{A}}$ receptors (Machado-Salas et al., 1977; Castel and Morris, 2000; Palomba et al., 2008) are consistent with the observed reduction in GABAergic current amplitude.

Compared with the deterioration of rhythmicity observed in the single-cell patch-clamp recordings, the deterioration of the ensemble electrical activity rhythms was less severe. The question arises how this difference can be explained. It was shown recently that SCN network interactions can compensate for genetically induced cellular deficits (Welsh et al., 2010) and that weak oscillators can produce profound rhythms when connected in a network (Ko et al., 2010). We found that, in the aged SCN, the FDR current and GABAergic activity changed at both phases of the cycle in the same direction, indicating that alterations at the single-cell level must have occurred because desynchronization alone cannot explain these findings. Our results lead us to suggest that aging affects primarily single-cell properties and that neuronal communication within the SCN network partially compensates for the deficits in cellular physiology.

\section{Sleep-wake recordings and behavioral patterns}

An unexpected finding was that the increase in $\tau$ and the decrease in total activity levels occurred much earlier in life ( $<300 \mathrm{~d}$ old $)$ than the change in $\alpha$ and $\rho$ and stabilizes above the age of $300 \mathrm{~d}$. The increment in $\tau$ has been associated with aging (Turek et al., 1995), but our findings suggest that, rather, these alterations occur during adulthood. The phase-shifting capacity of old animals was reduced to $83 \%$ of the capacity of young animals, consistent with previous studies (Biello, 2009).

We observed an increment in $\rho$ when animals were $\sim 700 \mathrm{~d}$ old. In animals $900 \mathrm{~d}$ old, $\rho$ was even longer, suggesting that this is a progressive phenomenon. Sleep-wake recordings confirmed that the shorter $\alpha$ in the older animals was not specific for wheelrunning behavior but was also apparent in the amount and distribution of waking. During the rest phase of nocturnal animals, SCN neuronal activity is high. When neuronal activity is more spread out over the day, $\rho$ increases (VanderLeest et al., 2007; Brown and Piggins, 2009). The observed increment in $\rho$ has im- plications for diurnal animals and humans. Because the activity and rest period of the cycle are coupled to the reverse parts of the SCN rhythm in diurnal species, the observed desynchrony of cellular oscillations would translate into an increase in $\alpha$ and a decrease in the sleep period in humans. This is in accordance with observations that aged humans sleep less (Dijk et al., 1999).

In the course of aging, an increment in fragmentation was observed in the behavioral activity patterns of old animals, which appeared independent of the decrease in the overall level of activity. Old animals also lost the ability to display long consolidated waking episodes. This resulted in less waking and more NREM sleep in the dark period. Thus, the behavioral activity pattern and the sleep-wake pattern were affected particularly during the subjective night. This corresponds with the phase of the cycle in which the major alterations were observed in the SCN network activity. The earlier onset of sleep at night corresponds also with the advance in the SCN electrical activity rhythms in old animals.

The findings suggest a tight relationship between alterations in SCN neuronal network activity and behavioral rhythm deterioration. The deterioration of the network may have followed from the changes in membrane and ionic channel properties, which were rather severe compared with the changes at the network level. One interpretation is that the aged network is, in fact, able to compensate for some cellular deficiencies but cannot rescue all deficits coming with age, leading to altered phase distributions within the SCN pacemaker. A better understanding of synchronization mechanisms within the SCN and the role of membrane events herein will provide a basis for interventions in clock-related diseases in the elderly.

\section{References}

Albus H, Vansteensel MJ, Michel S, Block GD, Meijer JH (2005) A GABAergic mechanism is necessary for coupling dissociable ventral and dorsal regional oscillators within the circadian clock. Curr Biol 15:886-893.

Aton SJ, Colwell CS, Harmar AJ, Waschek J, Herzog ED (2005) Vasoactive intestinal polypeptide mediates circadian rhythmicity and synchrony in mammalian clock neurons. Nat Neurosci 8:476-483.

Belle MD, Diekman CO, Forger DB, Piggins HD (2009) Daily electrical silencing in the mammalian circadian clock. Science 326:281-284.

Biello SM (2009) Circadian clock resetting in the mouse changes with age. Age (Dordr) 31:293-303.

Brown TM, Piggins HD (2007) Electrophysiology of the suprachiasmatic circadian clock. Prog Neurobiol 82:229-255.

Brown TM, Piggins HD (2009) Spatiotemporal heterogeneity in the electrical activity of suprachiasmatic nuclei neurons and their response to photoperiod. J Biol Rhythms 24:44-54.

Cai SQ, Sesti F (2009) Oxidation of a potassium channel causes progressive sensory function loss during aging. Nat Neurosci 12:611-617.

Castel M, Morris JF (2000) Morphological heterogeneity of the GABAergic network in the suprachiasmatic nucleus, the brain's circadian pacemaker. J Anat 196:1-13.

Chee CA, Roozendaal B, Swaab DF, Goudsmit E, Mirmiran M (1988) Vasoactive intestinal polypeptide neuron changes in the senile rat suprachiasmatic nucleus. Neurobiol Aging 9:307-312.

Deboer T, Fontana A, Tobler I (2002) Tumor necrosis factor (TNF) ligand and TNF receptor deficiency affects sleep and the sleep EEG. J Neurophysiol 88:839-846.

Deboer T, Ruijgrok G, Meijer JH (2007) Short light-dark cycles affect sleep in mice. Eur J Neurosci 26:3518-3523.

Dijk DJ, Duffy JF, Riel E, Shanahan TL, Czeisler CA (1999) Ageing and the circadian and homeostatic regulation of human sleep during forced desynchrony of rest, melatonin and temperature rhythms. J Physiol 516:611-627.

Duncan MJ, Herron JM, Hill SA (2001) Aging selectively suppresses vasoactive intestinal peptide messenger RNA expression in the suprachiasmatic nucleus of the Syrian hamster. Brain Res Mol Brain Res 87:196-203.

Duncan MJ, Hester JM, Hopper JA, Franklin KM (2010) The effects of aging 
and chronic fluoxetine treatment on circadian rhythms and suprachiasmatic nucleus expression of neuropeptide genes and 5-HT1B receptors. Eur J Neurosci 31:1646-1654.

Eilers PH (2003) A perfect smoother. Anal Chem 75:3631-3636.

Erraji-Benchekroun L, Underwood MD, Arango V, Galfalvy H, Pavlidis P, Smyrniotopoulos P, Mann JJ, Sibille E (2005) Molecular aging in human prefrontal cortex is selective and continuous throughout adult life. Biol Psychiatry 57:549-558.

Harper DG, Stopa EG, Kuo-Leblanc V, McKee AC, Asayama K, Volicer L, Kowall N, Satlin A (2008) Dorsomedial SCN neuronal subpopulations subserve different functions in human dementia. Brain 131:1609-1617.

Hofman MA, Swaab DF (2006) Living by the clock: the circadian pacemaker in older people. Ageing Res Rev 5:33-51.

Hurd MW, Ralph MR (1998) The significance of circadian organization for longevity in the golden hamster. J Biol Rhythms 13:430-436.

Itri JN, Michel S, Vansteensel MJ, Meijer JH, Colwell CS (2005) Fast delayed rectifier potassium current is required for circadian neural activity. Nat Neurosci 8:650-656.

Itri JN, Vosko AM, Schroeder A, Dragich JM, Michel S, Colwell CS (2010) Circadian regulation of a-type potassium currents in the suprachiasmatic nucleus. J Neurophysiol 103:632-640.

Itri J, Michel S, Waschek JA, Colwell CS (2004) Circadian rhythm in inhibitory synaptic transmission in the mouse suprachiasmatic nucleus. J Neurophysiol 92:311-319.

Ko CH, Yamada YR, Welsh DK, Buhr ED, Liu AC, Zhang EE, Ralph MR, Kay SA, Forger DB, Takahashi JS (2010) Emergence of noise-induced oscillations in the central circadian pacemaker. PLoS Biol 8:e1000513.

Kuhlman SJ, McMahon DG (2004) Rhythmic regulation of membrane potential and potassium current persists in SCN neurons in the absence of environmental input. Eur J Neurosci 20:1113-1117.

Liu C, Reppert SM (2000) GABA synchronizes clock cells within the suprachiasmatic circadian clock. Neuron 25:123-128.

Luebke JI, Chang YM (2007) Effects of aging on the electrophysiological properties of layer 5 pyramidal cells in the monkey prefrontal cortex. Neuroscience 150:556-562.

Luebke J, Barbas H, Peters A (2010) Effects of normal aging on prefrontal area 46 in the rhesus monkey. Brain Res Rev 62:212-232.

Machado-Salas J, Scheibel ME, Scheibel AB (1977) Morphologic changes in the hypothalamus of the old mouse. Exp Neurol 57:102-111.

Mahlberg R, Tilmann A, Salewski L, Kunz D (2006) Normative data on the daily profile of urinary 6-sulfatoxymelatonin in healthy subjects between the ages of 20 and 84. Psychoneuroendocrinology 31:634-641.

Michel S, Itri J, Colwell CS (2002) Excitatory mechanisms in the suprachiasmatic nucleus: the role of AMPA/KA glutamate receptors. J Neurophysiol 88:817-828.

Nakamura TJ, Nakamura W, Yamazaki S, Kudo T, Cutler T, Colwell CS, Block GD (2011) Age-related decline in circadian output. J Neurosci 31:10201-10205.

Nygård M, Palomba M (2006) The GABAergic network in the suprachiasmatic nucleus as a key regulator of the biological clock: does it change during senescence? Chronobiol Int 23:427-435.
Nygård M, Hill RH, Wikström MA, Kristensson K (2005) Age-related changes in electrophysiological properties of the mouse suprachiasmatic nucleus in vitro. Brain Res Bull 65:149-154.

Palomba M, Nygård M, Florenzano F, Bertini G, Kristensson K, Bentivoglio M (2008) Decline of the presynaptic network, including GABAergic terminals, in the aging suprachiasmatic nucleus of the mouse. J Biol Rhythms 23:220-231.

Satinoff E, Li H, Tcheng TK, Liu C, McArthur AJ, Medanic M, Gillette MU (1993) Do the suprachiasmatic nuclei oscillate in old rats as they do in young ones? Am J Physiol 265:R1216-R1222.

Schaap J, Albus H, VanderLeest HT, Eilers PH, Détári L, Meijer JH (2003) Heterogeneity of rhythmic suprachiasmatic nucleus neurons: Implications for circadian waveform and photoperiodic encoding. Proc Natl Acad Sci U S A 100:15994-15999.

Smit DJ, Boersma M, van Beijsterveldt CE, Posthuma D, Boomsma DI, Stam CJ, de Geus EJ (2010) Endophenotypes in a dynamically connected brain. Behav Genet 40:167-177.

Stam CJ, Montez T, Jones BF, Rombouts SA, van der Made Y, Pijnenburg YA, Scheltens P (2005) Disturbed fluctuations of resting state EEG synchronization in Alzheimer's disease. Clin Neurophysiol 116:708-715.

Swaab DF, Fliers E, Fisser B (1985) The vasopressin containing neurons in the human brain; changes during ageing and senile dementia. Br J Clin Pract Suppl 39:7-10.

Takahashi JS, Shimomura K, Kumar V (2008) Searching for genes underlying behavior: lessons from circadian rhythms. Science 322:909-912.

Turek FW, Penev P, Zhang Y, van Reeth O, Zee P (1995) Effects of age on the circadian system. Neurosci Biobehav Rev 19:53-58.

VanderLeest HT, Houben T, Michel S, Deboer T, Albus H, Vansteensel MJ, Block GD, Meijer JH (2007) Seasonal encoding by the circadian pacemaker of the SCN. Curr Biol 17:468-473.

VanderLeest HT, Rohling JH, Michel S, Meijer JH (2009) Phase shifting capacity of the circadian pacemaker determined by the SCN neuronal network organization. PLoS One 4:e4976.

Vyazovskiy VV, Ruijgrok G, Deboer T, Tobler I (2006) Running wheel accessibility affects the regional electroencephalogram during sleep in mice. Cereb Cortex 16:328-336.

Watanabe A, Shibata S, Watanabe S (1995) Circadian rhythm of spontaneous neuronal activity in the suprachiasmatic nucleus of old hamster in vitro. Brain Res 695:237-239.

Welsh DK, Logothetis DE, Meister M, Reppert SM (1995) Individual neurons dissociated from rat suprachiasmatic nucleus express independently phased circadian firing rhythms. Neuron 14:697-706.

Welsh DK, Takahashi JS, Kay SA (2010) Suprachiasmatic nucleus: cell autonomy and network properties. Annu Rev Physiol 72:551-577.

Witting W, Kwa IH, Eikelenboom P, Mirmiran M, Swaab DF (1990) Alterations in the circadian rest-activity rhythm in aging and Alzheimer's disease. Biol Psychiatry 27:563-572.

Wu YH, Zhou JN, Van Heerikhuize J, Jockers R, Swaab DF (2007) Decreased MT1 melatonin receptor expression in the suprachiasmatic nucleus in aging and Alzheimer's disease. Neurobiol Aging 28:1239-1247. 